Für behandlungsbedürftige Hämangiome, die eine maximale Tiefenausdehnung von 3-5 mm und eine Flächenausdehnung von höchstens $2 \mathrm{~cm}$ Durchmesser aufweisen, ist die Standardbehandlung eine Kontakt-Kryotherapie bei $-32^{\circ} \mathrm{C}$ (Abb. 6). Geeignet sind bei entsprechender klinischer Erfahrung alle Lokalisationen, sagte Schneider. Die Anwendung sollte unter Lokalanästhesie mit einer Applikationszeit von 15-20 Sekunden durchgeführt werden.

Bei größeren Hämangiomen an kritischen Lokalisationen ist heute systemisch verabreichtes Propranolol Mittel der Wahl. Dies ist zugelassen für proliferative infantile Hämanagiome ab der fünften Lebenswoche, die funktionsbeeinträchtigend oder lebensbedrohlich sind, die mit schmerzhaften Ulzerationen einhergehen oder bei denen die Gefahr von bleibenden Narben oder Entstellungen besteht. Die Behandlungsdauer beträgt in der Regel 6 Monate, abhängig vom individuellen Behandlungsverlauf. Vor Therapiebeginn sollte ein EKG durchgeführt werden, Herzfrequenz und Blutdruck sollten überwacht
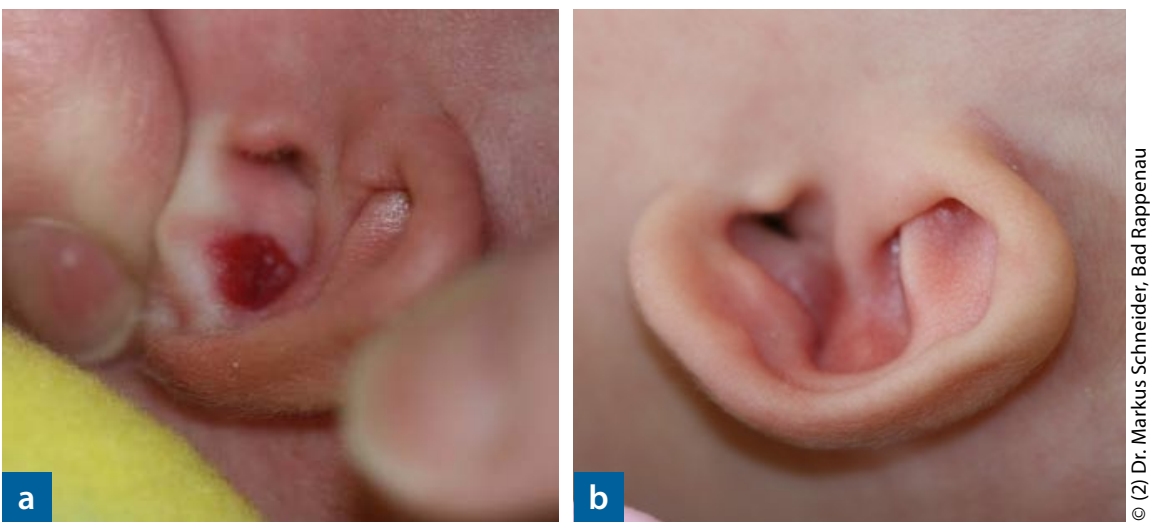

Abb. 6: Hämangiom vor (a) und einen Monat nach Kryotherapie (b)

werden. Verbleibende Restbefunde können mit dem Laser behandelt werden. Bei therapiebedürftigen Hämagiomen, die für eine Kryotherapie zu ausgeprägt sind, für die aber (noch) keine Indikation für eine systemische Behandlung vorliegt, kann ein individueller Heilversuch mit topischem Propranolol in Frage kommen, sagte Schneider. Einsatzgebiete sind insbesondere Hämangiome im Gluteal- und Genitalbereich oder an Händen und Füßen. Die Behandlungs- dauer beträgt in der Regel 12 Wochen. Relevante Nebenwirkungen sind unter der Therapie bislang nicht aufgetreten. Standardisierte Zubereitungen und einheitliche Anwendungsschemata stehen allerdings noch nicht zur Verfügung, Zulassungsstudien werden mit Spannung erwartet. Angelika Bauer-Delto

Schneider M. Aktuelles zu infantilen Hämangiomen

\title{
Akne: Nicht nur in der Pubertät
}

A kne zählt bei Jugendlichen zu den häufigsten Hauterkrankungen. Treten solche Hauterscheinungen im Kleinkindalter auf, wird oft nicht an eine Akne gedacht, sagte PD Dr. Christina Schnopp, Hautärztin in München. Eine korrekte Diagnose und Therapie sei jedoch wichtig, da ein hohes $\mathrm{Ri}$ siko für Narbenbildungen besteht.

Die Acne infantum (Abb. 7) betrifft typischerweise Kinder zwischen dem 6. und 13. Lebensmonat und zeigt das klassische Bild einer Akne mit offenen und geschlossenen Komedonen, entzündlichen Papulopusteln und manchmal auch knotigen und zystischen Läsionen. Lokalisationen sind vor allem die Wangen, eventuell auch der Kinnbereich.

Endokrinologische Störungen sind in der Regel nicht assoziiert. Bestehen keine weiteren klinischen Hinweise auf eine Hyperandrogenämie, sei eine routinemäßige hormonelle Abklärung nicht erforderlich, erklärte Schnopp.
Die Acne infantum wird wie eine $\mathrm{Pu}$ bertätsakne behandelt. Sicherheit und Wirksamkeit von Akne-Medikamenten sind allerdings bei Kleinkindern nicht untersucht, so Schnopp. Die Dosierung sollte niedriger gewählt werden als bei Jugendlichen. Topisch eingesetzt werden Benzoylperoxid, Adapalen und Zinkoxid, eventuell in Kombination mit Erythromycin. Auch systemisches Erythromycin kommt in Frage. Den Eltern ist anzuraten, fettreiche Externa zu vermeiden. In sehr schweren Fällen kann Isotretinoin unter engmaschiger Kontrolle möglicher Nebenwirkungen gegeben werden. Die Dosierung sollte bei $0,5 \mathrm{mg}$ /kg Körpergewicht liegen; dies sei jedoch eine Herausforderung, da entsprechende Darreichungsformen nicht zur Verfügung stehen, räumte Schnopp ein.

Die Pubertätsakne tritt heute zunehmend früher auf, so die Erfahrungen von Pädiatern und Dermatologen. Eine ausgeprägte Akne vor dem 11. Lebens-

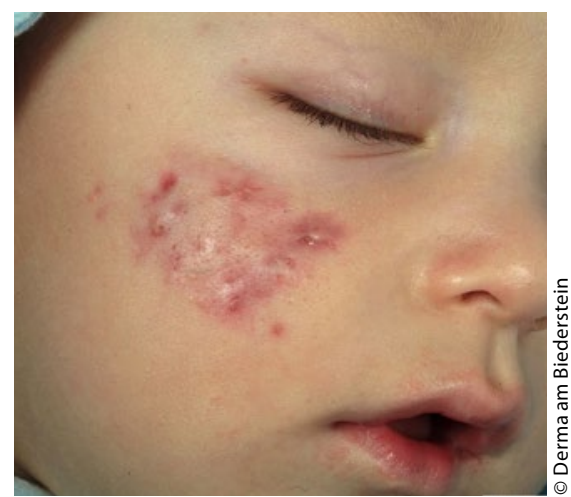

Abb. 7: Acne infantum

jahr sollte jedoch stets Anlass für eine hormonelle Abklärung sein, betonte Schnopp. Zudem sollten Knochenalter und Wachstumskurve überprüft werden. Insbesondere ist an ein zugrunde liegendes adrenogenitales Syndrom, adrenale und gonadale Tumoren sowie polyzystische Ovarien zu denken.

Angelika Bauer-Delto

Schnopp C. Akne bei Kindern und Jugendlichen 